Hydrogen bonding may account for the better results obtained by Human and Mills when they used pyridine in one molecular proportion in their experiments with carboxylic acids (III).

W. Gerrard

Northern Polytechnic,

K. H. V. FrenoH

Holloway Road,

London, N.7.

Jan. 13.

${ }^{1}$ Human and Mills, Nature, 158, 877 (1946).

Darzens, C.R. Acad. Sci., Paris, 152, 1314, 1601 (1911)

- Gerrard, J. Chem. Soc., 688 (1936); 99 (1939); 218 (1940); 85 (1944).

\section{Absolute Values of the Rates of Unimolecular Reactions}

EXPERLMENTAL unimolecular reaction-rates in gases are adequately represented by the equation :

$$
K_{\mathrm{uni}} \equiv-\frac{1}{N} \frac{d N}{d t}=a \exp (-E / k T) \mathrm{sec}^{-1},
$$

where $N$ is the concentration, $T$ the absolute temperature, and $k$ is Boltzmann's constant. 'The fact that $a$ is in most cases found to be of the order of magnitude of intramolecular vibration frequencies $\left(10^{14} \mathrm{sec}^{-1}\right)$ has been much discussed. By an approach akin to that of Polanyi and Wigner ${ }^{1}$, I obtained a theoretical reaction-rate in which $a$, although not precisely determined, was of this order of magnitude $e^{2,3}$. This result can now be made precise.

The gas molecule is treated (as before) as a classical system with $n$ internal degrees of freedom, and $n$ normal modes of harmonic vibration; the vibration frequencies $v_{1}, \ldots v_{n}$ of these modes are assumed to be linearly independent numbers. The timevariation of a geometrical co-ordinate $q$, such as the extension of an inter-atomic bond, is a sum of normalmode terms :

$$
q=\sum_{8=1}^{n} \alpha_{8} \sqrt{\varepsilon_{8}} \cos 2 \pi\left(\nu_{8} t+\varphi_{8}\right),
$$

where $\varepsilon_{8}$ is the energy and $\varphi_{8}$ the phase-angle in the $s$ th normal mode; on this classical picture, the $\varepsilon_{8}$ and $\varphi_{s}$ change only at molecular collisions. The 'amplitude factors' $\alpha_{8}$ are constants characteristic of the particular co-ordinate $q$ considered.

The molecule is assumed to dissociate when one particular co-ordinate $q$ attains a critical high value $q_{0}$. This value is attainable only after collision has raised the internal energy to satisfy

$$
\Sigma \alpha_{8} \sqrt{\varepsilon_{8}} \geqq q_{0},
$$

but the molecule dissociates only if the terms in (2) have time to come sufficiently into phase for $q$ to reach $q_{0}$ before further collision removes the high energy. If then $L\left(\varepsilon_{1}, \ldots \varepsilon_{n} ; q_{0}\right)$ is the frequency with which the sum (2) would attain $q_{0}$ if undisturbed, $L$ may be taken to represent the proportion of molecules with energies $\left(\varepsilon_{1}, \ldots \varepsilon_{n}\right)$ which dissociate per second.

At the high pressures at which de-energizing collisions are much more frequent than dissociations, the number of molecules with energies in the ranges $\left(\varepsilon_{1}, \varepsilon_{1}+d \varepsilon_{1}\right) \ldots\left(\varepsilon_{n}, \varepsilon_{n}+d \varepsilon_{n}\right)$ is very nearly the equilibrium number, namely,

$$
N(k T)^{-n} \exp \left(-\Sigma \varepsilon_{8} / k T\right) d \varepsilon_{1} \ldots d \varepsilon_{n} .
$$

A fraction $L$ of these dissociate per second; thus the total dissociation-rate is the first-order rate :

$$
\begin{array}{r}
K_{\mathrm{uni}} \equiv-\frac{1}{N} \frac{d N}{d t}=\int_{0}^{\infty} \ldots \int_{0}^{\infty} L\left(\varepsilon_{1}, \ldots \varepsilon_{n} ; q_{0}\right) \\
(k T)^{-n} \exp \left(-\Sigma \varepsilon_{s} / k T\right) d \varepsilon_{1} \ldots d \varepsilon_{n} .
\end{array}
$$

(We can integrate from $\varepsilon_{g}=0$, since $L=0$ where (3) is not satisfied.)

Now

$$
L=\frac{1}{2} \lim _{x \rightarrow \infty}\{M(\tau) / \tau\},
$$

where $M(\tau)$ is the number of zeros, for $0 \leqq t<\tau$, of $q-q_{0}$ with $q$ defined by (2). I used before ${ }^{2}$ an approximate formula for this limit, but use now a formula due to $\mathrm{Kac}^{4}$, which gives in our notation:

$$
\begin{aligned}
& L\left(\varepsilon_{1}, \ldots \varepsilon_{n} ; q_{0}\right)=\frac{1}{4 \pi^{2}} \int_{-\infty}^{\infty} \int_{-\infty}^{\infty} \frac{\cos \left(q_{0} \xi\right)}{\eta^{2}}\left\{\prod_{1}^{n} J_{0}\left(\xi \alpha_{8} \sqrt{\varepsilon_{8}}\right)-\right. \\
& \left.\prod_{1}^{n} J_{0}\left[\alpha_{8} \sqrt{\varepsilon_{8}\left(\xi^{2}+4 \pi^{2} v_{8}{ }^{2} \eta^{2}\right)}\right]\right\} d \xi d \eta \text {. }
\end{aligned}
$$

Inserting this in (4) and integrating first for the $\varepsilon_{8}$ by means of Weber's formula ${ }^{5}$, we find that

$$
K_{\text {uni }}=\nu \exp \left(-q_{0}^{2} / \alpha^{2} k T\right) \text {, }
$$

where

$$
\alpha^{2}=\Sigma \alpha_{8}^{2}, \quad \nu^{2}=\alpha^{-2} \Sigma \alpha_{8}^{2} v_{8}^{2} .
$$

It is easily found that the minimum total internal energy for dissociation, that is, the minimum $\Sigma \varepsilon_{8}$ satisfying (3), is

Thus, finally,

$$
E_{0}=q_{0}^{2} / \alpha^{2}
$$

$$
K_{\text {uni }}=\nu \exp \left(-E_{0} / k T\right) .
$$

The factor $\nu$ in this result is, by (6), the root mean square of the vibration frequencies weighted by the amplitude factors $\alpha_{8}$. It is thus independent of temperature, so that ${ }^{3}$ the energy $E_{0}$ in (7) may be identified with the empirical $E$ of (1) as the 'activation energy'.

A full discussion of this theory, with an extension to low-pressure dissociation-rates, will be given elsewhere.

Department of Mathematics,

\section{N. B. Slater}

\section{University, Leeds.} Dec. 27.

${ }^{1}$ Polanyi and Wigner, Z. phys. Chem., A, 139, 439 (1928).

- Slater, Proc. Camb. Phil. Soc., 35, 56 (1939).

"Fowler and Guggenheim, "Statistical Thermodynamics", 529 (1939).

Kac, Amer. J. Math., 65, 609 (1943).

s Watson, "Theory of Bessel Functions", 393 (1922).

\section{Crystal Structures of 2-Amino-4-Methyl-6- Chloropyrimidine and 2-Amino-4,6-Dichloro- pyrimidine}

A SERIES of investigations on the structures of pyrimidines and purines is being carried out in close association with the work of Prof. A. R. Todd and his collaborators on the chemistry of these and related compounds.

No previous structural work on pyrimidines has been reported in the literature, so an extensive search was made for compounds which seemed likely to permit of a determination of structure without previous assumptions as to the nature of the pyrimidine molecule. Two isomorphous pyrimidines were selected : 2-amino-4-methyl-6-chloropyrimidine (I) and 2-amino-4,6-dichloropyrimidine (II). 